Portland State University

PDXScholar

$5-25-2018$

\title{
Optimization of Carbon Supported Palladium and Gold Nanoparticles for Hydrodechlorination
}

Candice Stauffer

Portland State University

Follow this and additional works at: https://pdxscholar.library.pdx.edu/honorstheses

Let us know how access to this document benefits you.

Recommended Citation

Stauffer, Candice, "Optimization of Carbon Supported Palladium and Gold Nanoparticles for Hydrodechlorination" (2018). University Honors Theses. Paper 563.

https://doi.org/10.15760/honors.570

This Thesis is brought to you for free and open access. It has been accepted for inclusion in University Honors Theses by an authorized administrator of PDXScholar. Please contact us if we can make this document more accessible: pdxscholar@pdx.edu. 
Optimization of Carbon Supported Palladium and Gold Nanoparticles for Hydrodechlorination

by

\title{
Candice Stauffer
}

An undergraduate honors thesis submitted in partial fulfillment of the

\author{
requirements for the degree of \\ Bachelor of Science \\ in \\ University Honors \\ and \\ Physics
}

Thesis Adviser

Dr. Jun Jiao

Portland State University 


\begin{abstract}
The contamination of groundwater by trichloroethylene (TCE) and other related contaminants is a compromise to the safety of drinking water all over the world. There have been many efforts to solve this issue, but all possible solutions have come at the cost of excessive waste or further contamination of the environment. The palladium $(\mathrm{Pd})$, gold $(\mathrm{Au})$ coated carbon supported catalyst makes use of current nanotechnology to provide an efficient method of degrading TCE to non-toxic levels of ethene and ethane. This thesis discusses the optimization of the novel solvothermal, green synthesis process of $\mathrm{Pd} / \mathrm{Au}$ carbon supported nanoparticles (NPs) devolved by the Jiao Group at Portland State University. The pre-sonication of Au precursor, washing of the carbon supported NP samples, and reaction time of the samples were explored. Evidence suggested that sonicating the Au precursor did not, alone, synthesize nanoparticles, washing the catalysts did provide an effective method of ensuring nanoparticles adhered to their support, and that a reaction time of down to an hour may be just as effective as a reaction time of 24 hours. The preliminary results gathered here are promising results to suggest the claims mentioned above but will require further investigation for a definitive confirmation.
\end{abstract}




\section{Table of Contents}

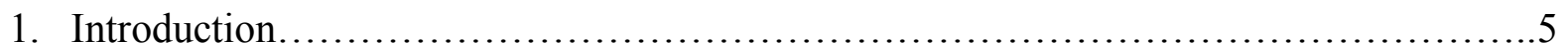

1.1 Groundwater Contamination and Current Methods for Their Removal.........5

1.2 Synthesis of Carbon Supported Pd/Au Nanoparticles......................... 7

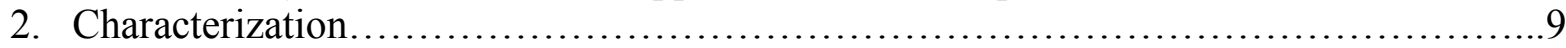

3. Experimental Procedures, Results, and Discussion................................. 10

3.1 Effect of Gold Precursor Sonication..................................... 10

3.2 Effect of Washing Pd/Au Catalyst......................................13

3.3 Effect of Synthesis Formation Time..................................... 18

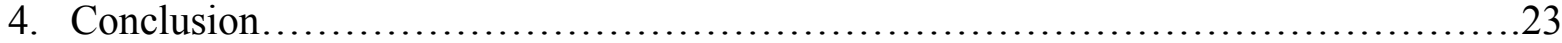

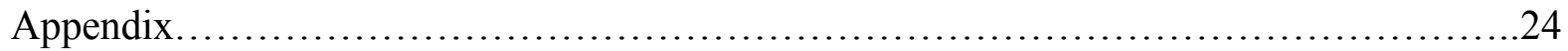

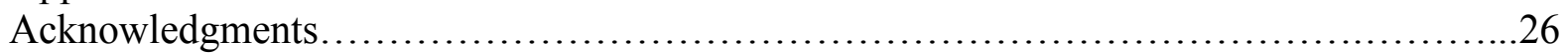

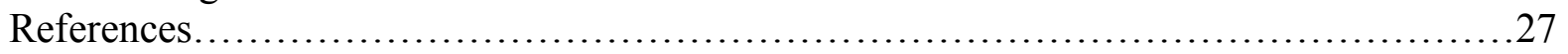




\section{List of Tables and Figures}

Figure 1: Mechanism for the degradation of TCE in the presence of Pd-based catalyst..........6

Figure 2: Degradation of TCE with GAC, Pd on GAC, Au on GAC, and Pd/Au NPs on GAC...7

Figure 3: An outline of the $\mathrm{Pd} / \mathrm{Au}$ NP catalyst schematic simplified into 6 steps................8

Figure 4: SEM image of $\mathrm{Pd} / \mathrm{Au}$ on the surface of GAC ................................. 10

Figure 5: Sigma-Aldrich Spectrums of Au Nanoparticles of different sizes...................12

Figure 6: Schematic of how the carbon supported $\mathrm{Pd} / \mathrm{Au}$ catalyst is washed....................14

Figure 7: Post-wash acetone spectrum............................................ 15

Figure 8: MATLAB Analysis of Post-Wash Acetone Spectrum........................... 16

Figure 9: Low magnification and high magnification TEM images of $\mathrm{Pd} / \mathrm{Au}$ on graphene......19

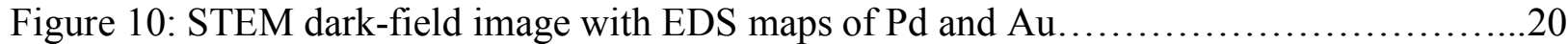

Figure 11: Low magnification TEM images of $\mathrm{Pd} / \mathrm{Au}$ NPs on graphene.....................20

Figure 12: UV-Vis analysis shows absorption is higher when NPs are given longer to react.....21

Table 1: Trials conducted to measure Au nanoparticles................................... 11

Table 2: Wavelength of light absorbed for each trial conducted and shown in Table 1..........12

Table 3: Where NPs would be expected if the existed in the leftover acetone solution...........16 


\section{Introduction}

\subsection{Groundwater Contamination and Current Methods for Their Removal}

Groundwater can be found almost everywhere and is supplied by rain and snowmelt that seeps down to aquifers made up of gravel, sand, sandstone, or fractured rock. $22 \%$ of the usable fresh water in the United States came from groundwater in 2010 while 50\% of people in the U.S. use groundwater as their primary drinking water [12] The largest use of groundwater is to irrigate crops that feed the U.S. population and livestock rendering it a vital natural resource especially for parts of the world that don't have abundant surface-water sources [12].

While aquifers are great natural water filters for physically larger impurities, they are still subject to the many chemicals that have increasingly been introduced into the environment. Common contaminants include hazardous waste, landfills, chemicals from dry cleaners, nitrates, road salts, atmospheric contaminants, and chemicals like Trichlorethylene (TCE) that are able to seep through the aquifers [7]. When consumed these contaminants can cause a variety of health issues from mild irritations to cancers and organ failure. An in-depth, multi-year study by the National Institute for Occupational Safety and Health expanded the evidence of groundwater contamination's danger by investigating an Oregonian company, Entek, and determining that employees who were exposed to TCE may have developed health problems more frequently than those who weren't [1][7].

To remove this harmful chemical, activated carbon filters are often used because of their highly adsorbent nature and ability to obtain harmful chemicals like TCE [3][8][9][10]. Activated carbon alone, however, can only remove the contaminants from the water. Once the contaminants are removed they are able to pollute other aspects of the environment, most concerningly, the air humans and animals breathe. TCE and many of the other known 
contaminates can be classified as volatile organic compounds (VOCs) and easily escape into the air from the surface of the carbon. In conclusion, activated carbon alone does not solve the contamination but transfers the toxin from one place to another.

The use of catalysts can be a highly effective method for the removal of hydrogenation, dehydrogenation, exchange, oxidation, reduction, and hydrogenolysis [3]. Palladium has been a well-known effective catalyst to provide an alternative reaction pathway from TCE to non-toxic ethene and ethane [3] (Figure 1).<smiles>ClC=CCl</smiles>

TCE
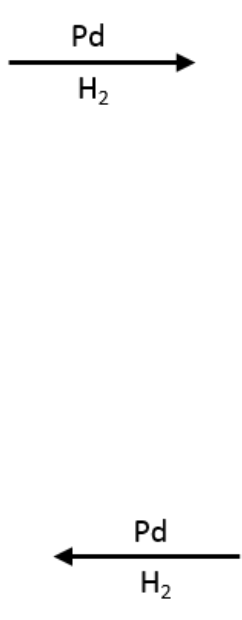

Ethane<smiles>ClC=CCl</smiles>

$1,1-D C E$<smiles>C=C</smiles>

Ethene<smiles>ClC=CCl</smiles>

cis-DCE

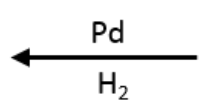<smiles>ClC=CCl</smiles>

Vinyl Chloride

Figure 1: Proposed mechanism for the degradation of TCE in the presence of Pd-based catalysts in a hydrogen atmosphere.

Manufacturing these catalysts and putting it into practice can be impractical for the industry, however. The Jiao group was able to synthesize a carbon supported Pd catalyst coated in Au that degraded TCE more quickly, sustainably, and efficiently than commercial carbon filters and Pd catalysts. The GC results show 2:1 Pd/Au catalyst degrad TCE more efficiently than other methods like $\mathrm{Pd}, \mathrm{Au}$, and GAC alone and is also supported by previous experiments 
$[8][9][10][13]$ (Figure 2). This paper investigates the catalyst and ways to optimize its synthesis, namely: (1) Pre-sonicating the Au precursor, (2) washing the $\mathrm{Pd} / \mathrm{Au}$ catalyst after the reaction to investigate the adherence of nanoparticles on their carbon support, (3) and the time at which the sample wasleft to react to synthesize these NPs.

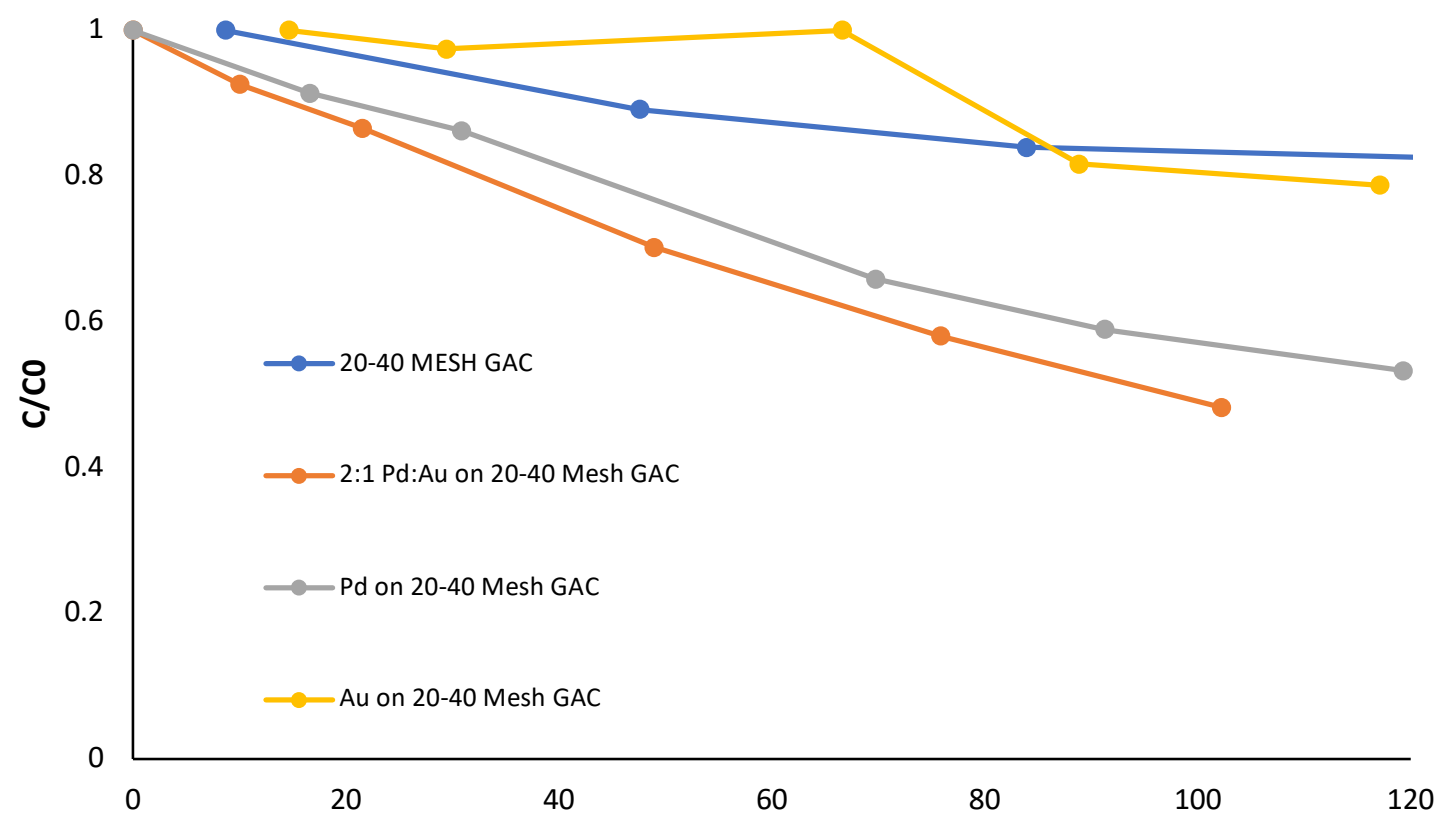

Figure 2: Degradation of TCE with GAC, Pd on GAC, Au on GAC, and Pd/Au NPs on GAC

\subsection{Synthesis of Pd Au Nanoparticles}

The method of Pd Au Nanoparticles as a catalyst supported on carbon is synthesized by the Jiao Group using a novel method that does not use any harsh chemicals or stabilizers. The asmade samples are designed to have a 2:1 Pd:Au molar ratio, and a $5 \mathrm{wt} \%$ of $\mathrm{Pd}$ on a carbon support, for comparison with corresponding a granular activated carbon (GAC) support. Typically, the synthesis of commercial GAC supported Pd catalysts and similar materials requires the use of complex multi-step processes - high temperatures; high pressures; [6], toxic chemicals such as sodium borohydride, [12] hydrazine, [12] and hydrogen peroxide; a pH control with alkaline or acidic components [11] — and some even require the use of surfactants 
and stabilizers. [2] Several of the methods used were achieved with this synthesis process, such as choosing safer chemical precursors, using safer solvents, and avoiding chemical derivatives.

Synthesis of the catalyst begins with the two metallic precursors Palladium (II) acetate (reagent grade, 98\%, Sigma-Aldrich) and Tetrachloroauric (III) acid trihydrate (Acros Organics) to produce $\mathrm{Pd}$ and Au nanoparticles. $4.5 \mathrm{mg}$ of Tetrachloroauric (III) acid trihydrate (Au Precursor) is first added to $1600 \mu \mathrm{L}$ of acetone. This solution is sonicated for 15 minutes. Meanwhile, $3 \mu \mathrm{L}$ of the Pd precursor is added to $20 \mathrm{~mL}$ of acetone. $800 \mu \mathrm{L}$ of the sonicated $\mathrm{Au}$ solution is added to the Pd solution. The combined solution is sonicated for 60 minutes. After 60 minutes the nanoparticles are removed from the solution and left to react with GAC (DARCO ${ }^{\text {, }}$ 20-40 mesh, Sigma-Aldrich) in a Teflon liner for up to 24 hours. Finally, the Pd/Au NPs supported on GAC are washed and dried. (Figure 3)

This method of synthesizing the $\mathrm{Pd} / \mathrm{Au}$ has been shown to be more effective than $\mathrm{Pd}$ catalysts, $\mathrm{Au}$, and commercial GAC alone [8][9][10][13].
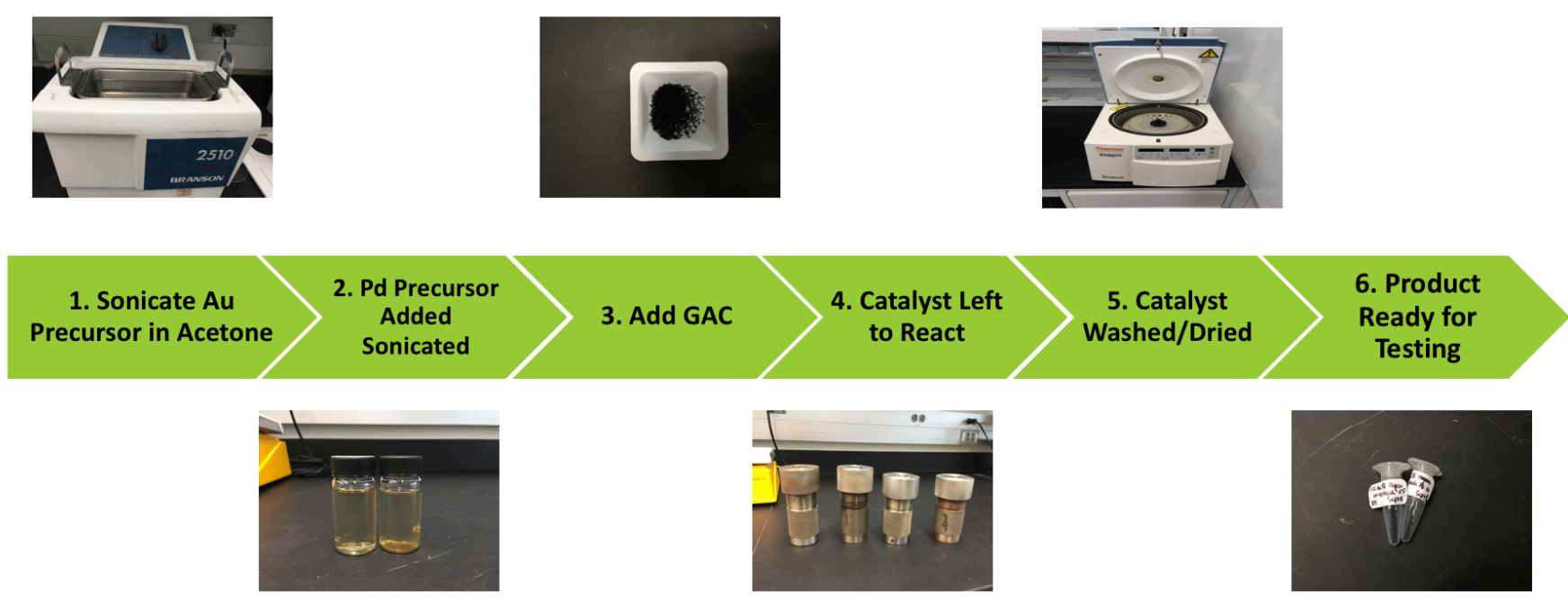

Figure 3: An outline of the Pd/Au NP catalyst schematic simplified into 6 steps. 


\section{Characterization}

An FEI Tecnai F20 Transmission Electron Microscope (TEM) equipped with scanning transmission electron microscopy (STEM) and energy dispersive X-ray spectroscopy (EDS) capabilities that operates at an accelerating voltage of $200 \mathrm{kV}$ and an FEI Sirion XL30 scanning electron microscope (SEM) with EDS and Shimadzu UV 3600 UV-Vis Spectrophotometer were used for the analysis of the catalyst. The reaction was monitored through headspace gas chromatography (GC) using an HP 5890 Series II GC equipped with a flame ionization detector (FID) and a DB-624 Agilent J\&W Scientific column (30 m long with $0.53 \mathrm{~mm}$ I.D.). 


\section{Experimental Procedures, Results, and Discussion}

\subsection{Effect of Gold Precursor Sonication}

Sonicating the Au precursor added several minutes to the synthesize process.

Investigating how the sonication effected the catalyst could save time in the synthesis process,

lead to an understanding of the strange unique affects between Pd and $\mathrm{Au}$, and an idea about how and when nanoparticles form.

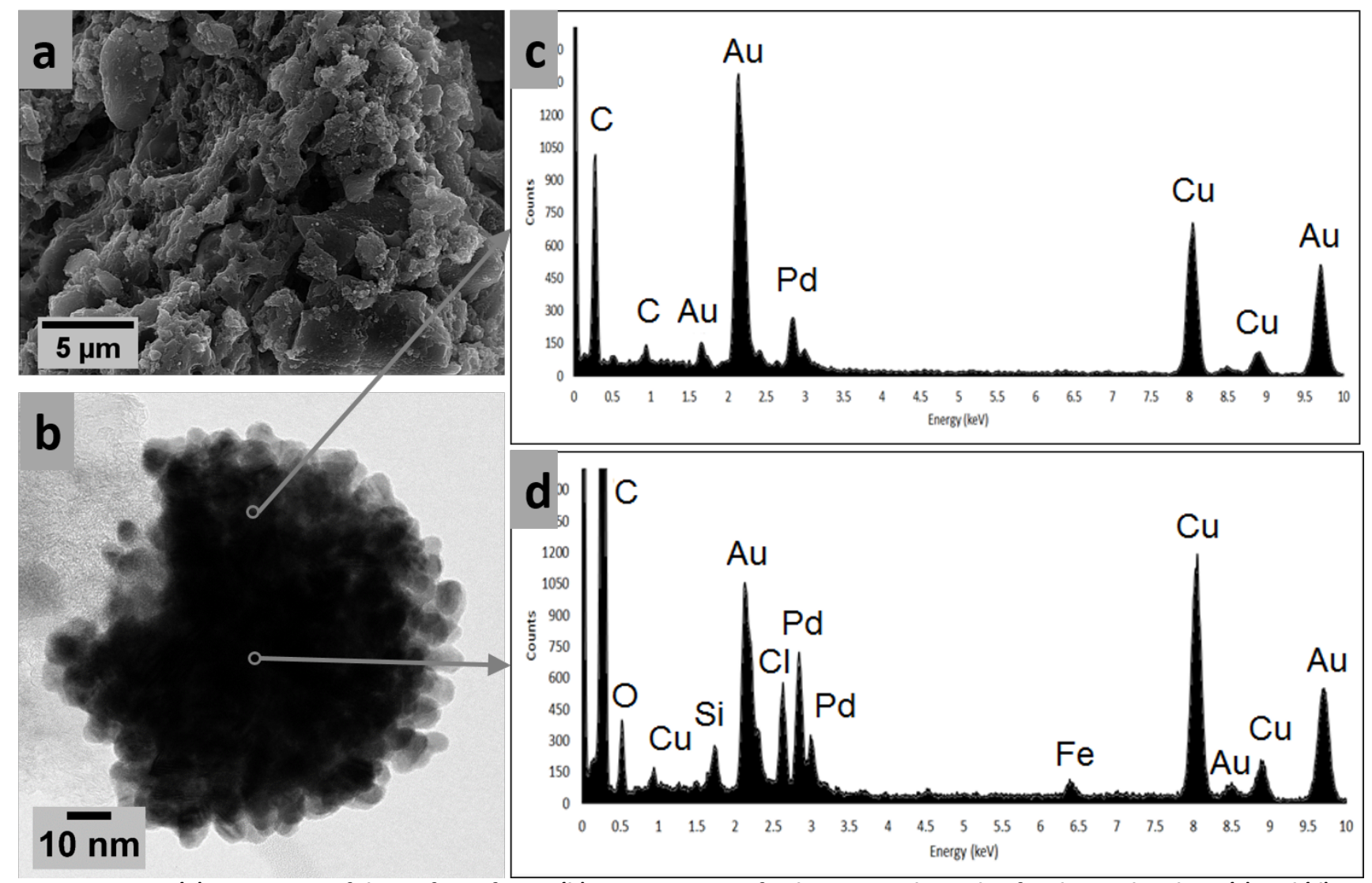

Figure 4: (a) SEM image of the surface of GAC. (b) HRTEM image of a dense particle made of carbon, Pd and Au. (c) and (d) are EDS spectra in the center and edge of the particle, showing dominant Au, and Pd peaks respectively

The objective of this experiment was to examine if pre-sonicating the Au precursor was the key step in synthesizing Au NPs. It would also help identify when in the synthesis process the Au NPs (Figure 4) were being formed. 
To achieve this experiment 6 trials were conducted to evaluate the first step in the Figure 3 schematic that varied the amount of time the Au precursor was sonicated, and amount of $\mathrm{Au}$ precursor added (Table 1).

Table 3: The 6 trials conducted that varied the amount of time the Au precursor was sonicated and the amount Au precursor that was added.

\begin{tabular}{|c|c|c|}
\hline Trial & Amount (mg) & Time (Min) \\
\hline Control 1 & 4.5 & 15 \\
\hline Trial 1 & 2.25 & 15 \\
\hline Trial 2 & 9.00 & 15 \\
\hline Trial 3 & 4.5 & 0 \\
\hline Trial 4 & 4.5 & 7.5 \\
\hline Trial 5 & 4.5 & 30 \\
\hline
\end{tabular}

The "Control Trial" were values used in previous experiments and literature [8][9][10][13]. Trials 1-5 varied the original amount of Au precursor or the original time the precursor was sonicated by a factor of 2 or $1 / 2$. The sonicated acetone and Au precursor solution was left to react for 60 min after it had been sonicated. Finally, the solution was observed using the UV-Vis spectrophotometer. The wavelength of light absorbed by each solution indicated whether or not it contained nanoparticles. The wavelength of light absorbed by Au nanoparticles is well-known throughout literature to be 515-572 nm depending on the diameter of the NP [3][4] (Figure 5). 


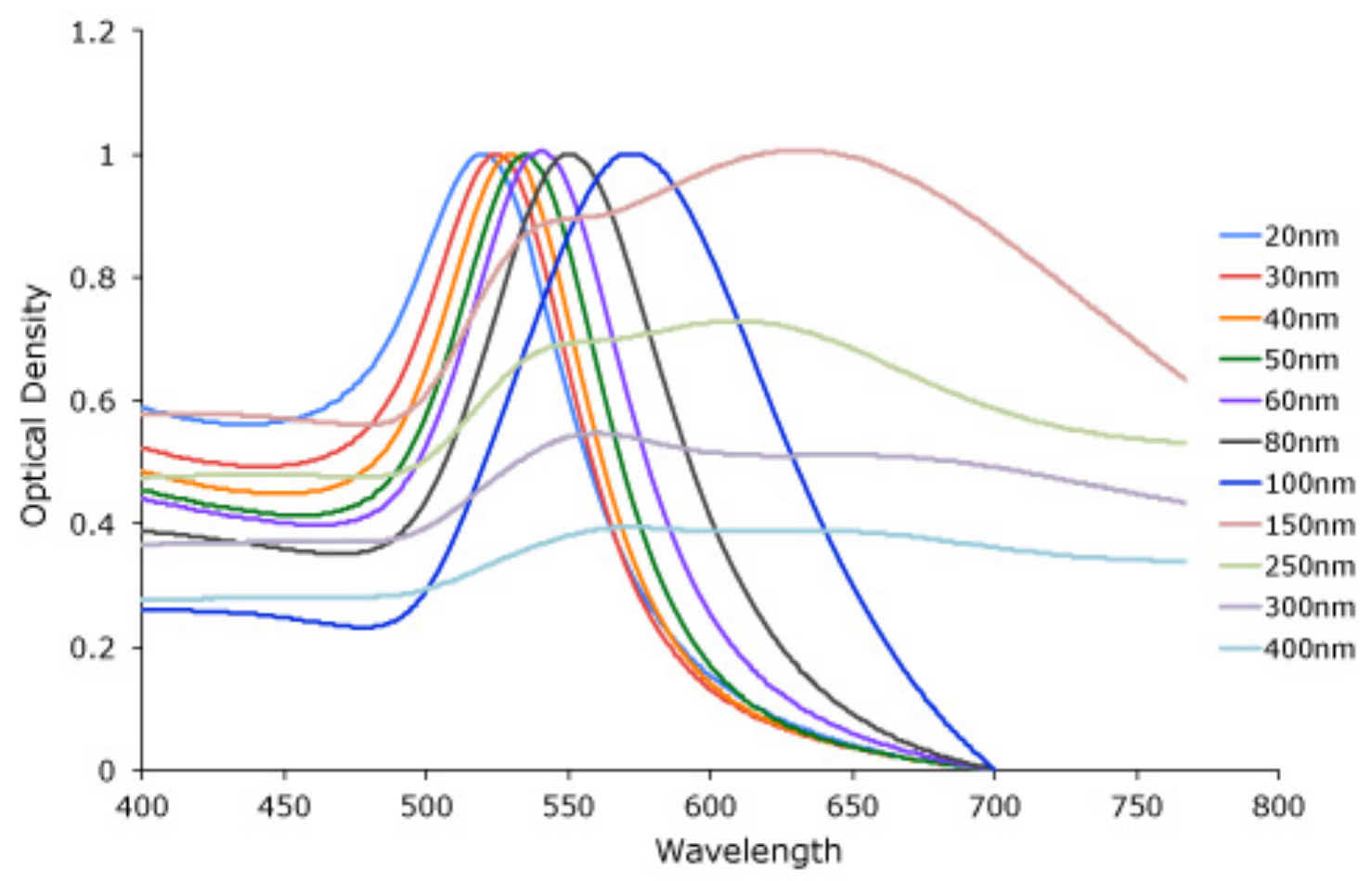

Figure 5 Spectrum of different sized Au nanoparticles (Sigma-Aldrich).

After synthesizing the samples for each trial in Table 1. Using the UV-Vis Spectrophotometer the wavelength of light at its peak absorbance of each Au sample in acetone was measured (Table 2).

Table 4: Wavelength of light absorbed for each trial conducted and shown in Table 1.

\begin{tabular}{|l|l|l|l|l|}
\hline Trial & Amount (Mg) & Time (Min) & Wavelength (nm) & Absorption \\
\hline Control & 4.5 & 15 & 395 & 0.962 \\
\hline Trial 1 & 2.25 & 15 & 361 & 1.924 \\
\hline Trial 2 & 9 & 15 & 414 & 2.047 \\
\hline
\end{tabular}




\begin{tabular}{|l|l|l|l|l|}
\hline Trial 3 & 4.5 & 0 & 397 & 2.014 \\
\hline Trial 4 & 4.5 & 7.5 & 409 & 2.033 \\
\hline Trial 5 & 4.5 & 30 & 399 & 2.021 \\
\hline
\end{tabular}

In the Control, the peak absorbance was recorded to be at $395 \mathrm{~nm}$, in Trial 1 the peak was at $361 \mathrm{~nm}$, in Trial 2 at $414 \mathrm{~nm}$, in Trial 3 at $397 \mathrm{~nm}$, in Trial 4 at $409 \mathrm{~nm}$ and in Trial 5 at 399 respectively.

None of the peaks matched the well-known wavelengths of light absorbed attributed to gold nanoparticles. Though it is confirmed that Au NPs do exist in the sample (Figure 4) it is unlikely that they form during the pre-sonication of Au. However, it is still possible that the presonication of Au contributes the ability of NPs to form during another part of the synthesis process. Speculations include that the sonication could break the gold chloride precursor into ions, allowing NPs to form later in the process when reacting with Pd NPs [5]. While synthesis of NPs are not observed immediately after the pre-sonication of Au here, the results should not be confirmed until another trial is conducted of acetone alone and compared with the results.

\subsection{Effect of Washing Pd/Au Catalyst}

The purpose of washing the $\mathrm{Pd} / \mathrm{Au}$ carbon supported catalyst is to guarantee that an excess of NPs is not present in the sample. Without washing the sample, leftover NPs could exist without fully adsorbing on to the surface of GAC. These excess particles could easily escape the surface of the carbon support and further contaminate the target medium. Here the spectrum of post-washed samples are studied to investigate whether or not any excess NPs are observed. 
Washing the $\mathrm{Pd} / \mathrm{Au}$ catalyst takes place in the $5^{\text {th }}$ step of the schematic in Figure 3 . An elaboration of the washing the samples, shown in Step 5 of Figure 3, incorporates the removal of acetone from the GAC supported Pd/Au NPs, then transferring the NPs on GAC to centrifuge tubes with clean acetone, and finally centrifuging the sample with an IEC Micromax RF centrifuge at 15000 RPM for 10 minutes to separate the sample from additional acetone. A more detailed schematic of Step 5 is shown in Figure 6.
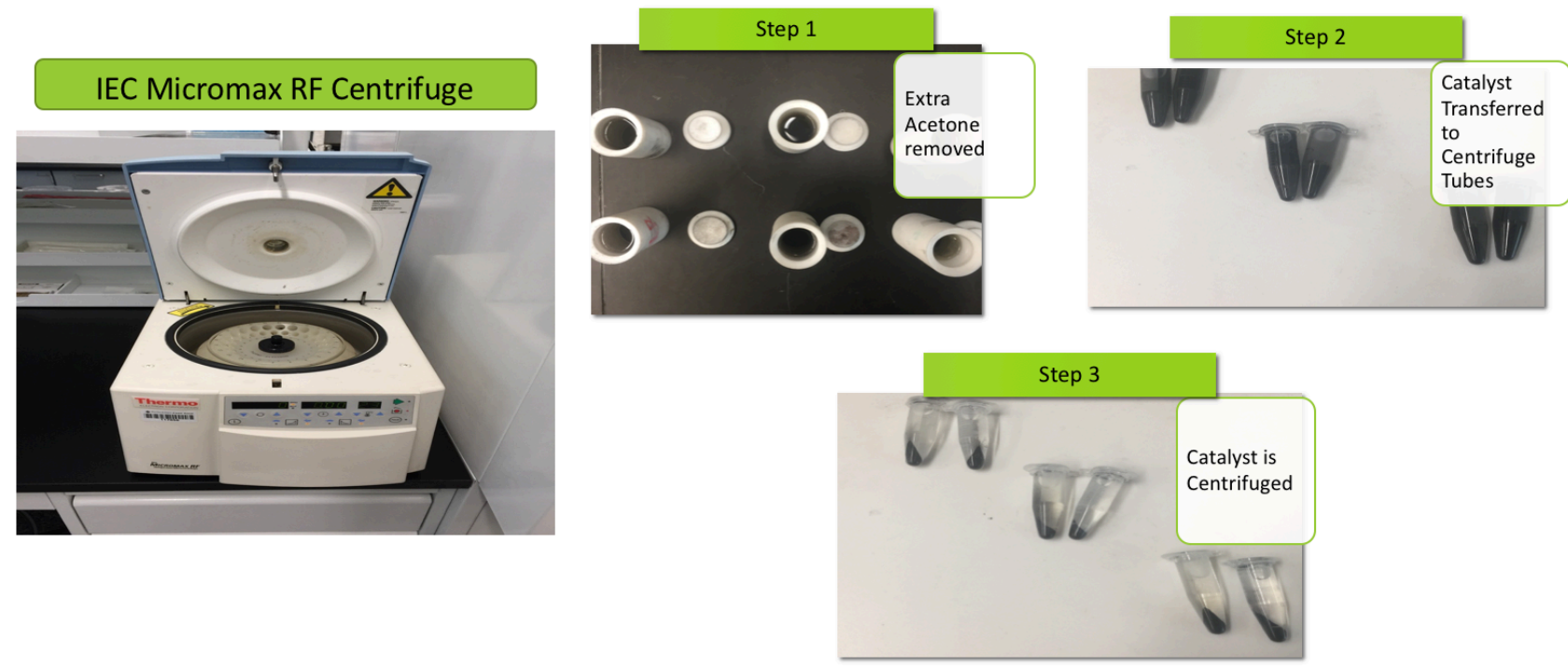

Figure 6: An outlined schematic of how the carbon supported Pd/Au catalyst is washed.

To perform this experiment, the wavelength of light absorbed from the leftover acetone "post-centrifuge" (shown in Step 3, Figure 6) is examined with the UV-Vis photo spectrometer using the same assumptions Au NPs in Part 4. If a wavelength of light is absorbed between 515$572 \mathrm{~nm}$ it could be possible that Au NPs are present in the leftover solution and thus have dislodged from the GAC surface.

The samples were washed an additional time to ensure that any nanoparticles that could be dislodged from the surface of the GAC would be dislodged. The wavelength of light absorbed is 
shown in Table 3 and plotted in Figure 7 and 8. Both Excel and MATLAB were used to compile and study the data collect.

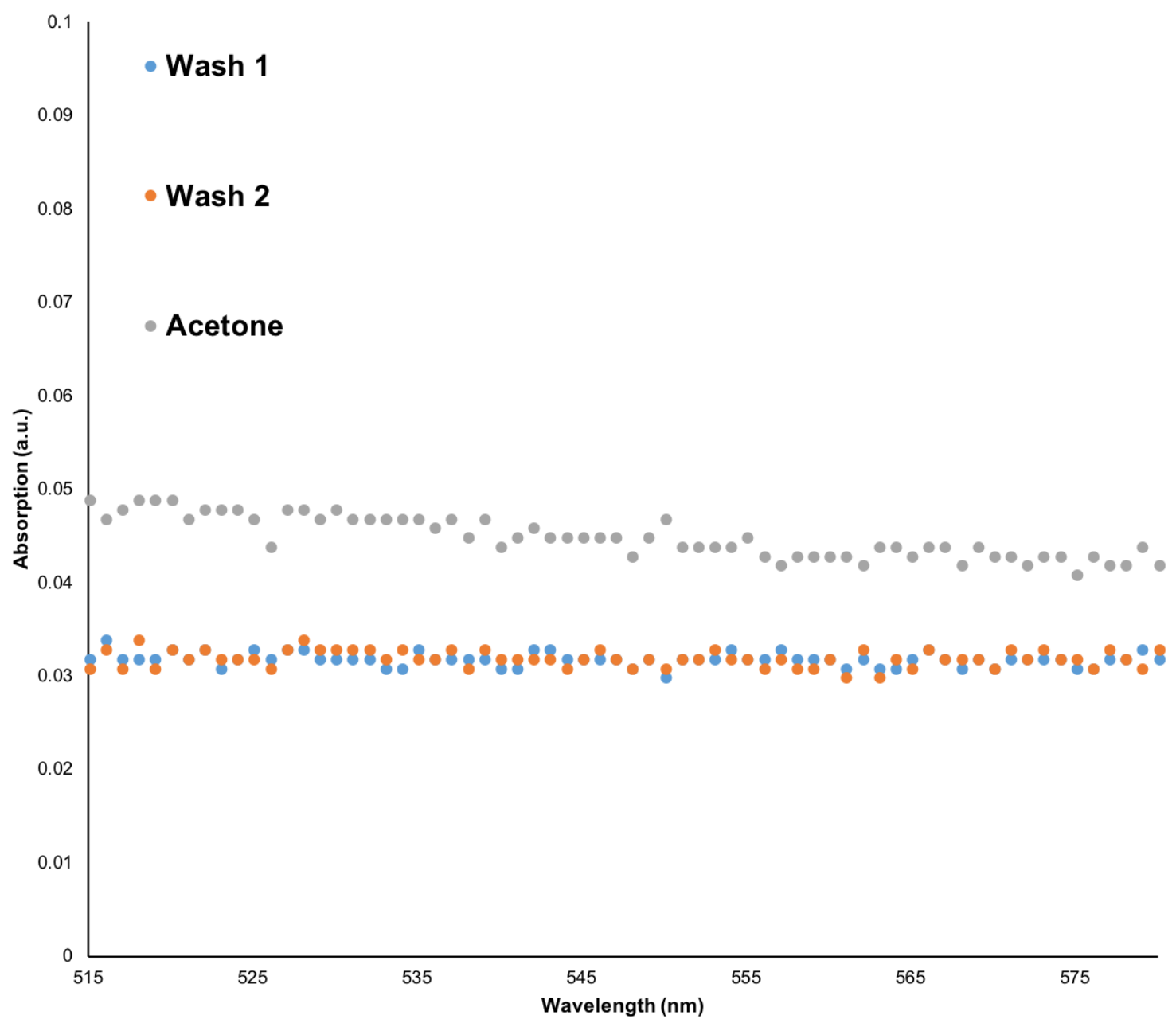

Figure 7: Spectrum of leftover acetone fluid where dislodged NPs would be expected to be found if they existed. The grey series shows pure acetone, while the blue and orange series show two washes of the same leftover acetone sample. While there does exist a small difference in absorption intensity, both washes show no signs of a peak in the absorption spectrum and very closely match the spectrum of acetone. 


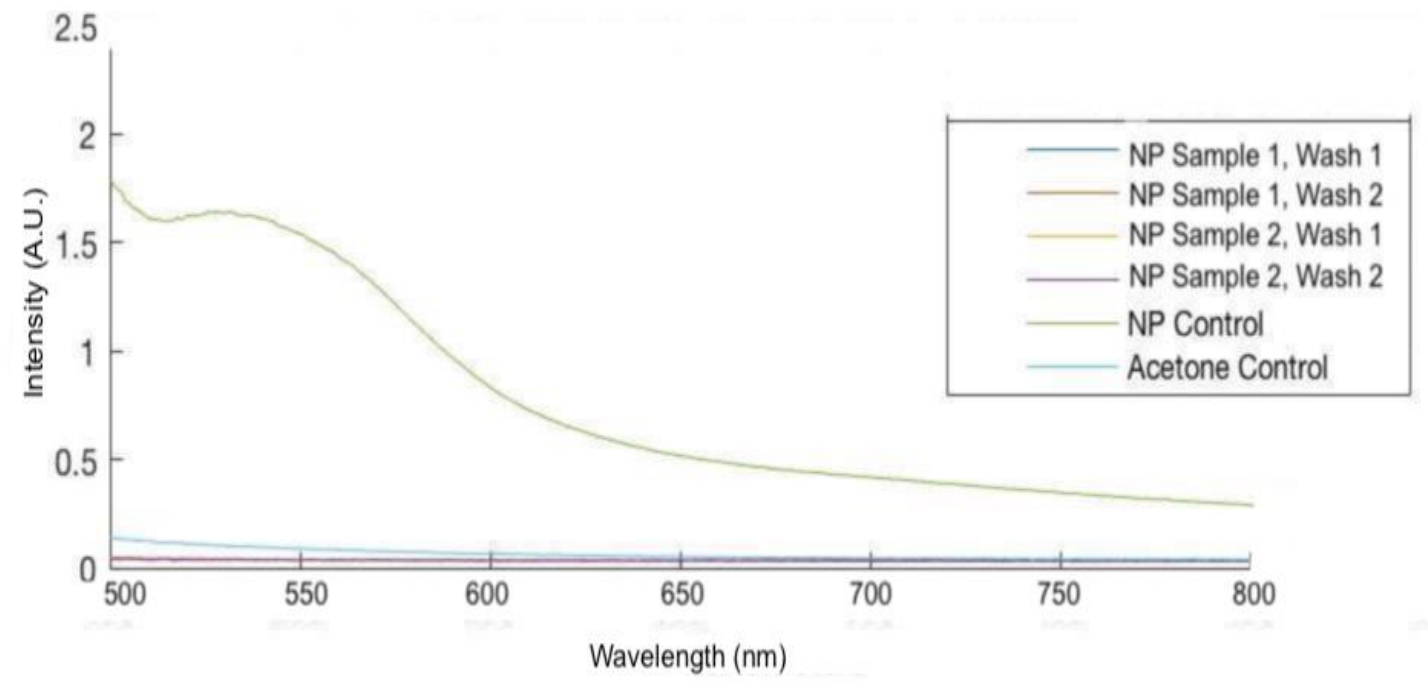

Figure 8: Data analyzed in Matlab comparing 2 samples and 2 controls

Table 5: An excerpt of the wavelength of light absorbed between a region where NPs would be expected if the existed in the leftover acetone solution.

\begin{tabular}{|r|r|r|r|}
\hline Wavelength & \multicolumn{1}{|l|}{ Wash 1 } & Wash 2 & Fresh \\
& Absorption & Absorption & Acetone \\
& (A.U.) & (A.U.) & Absorption \\
& & & (A.U.) \\
\hline 525 & 0.058 & 0.049 & 0.047 \\
\hline 526 & 0.056 & 0.046 & 0.044 \\
\hline 527 & 0.058 & 0.05 & 0.048 \\
\hline 528 & 0.057 & 0.049 & 0.048 \\
\hline 529 & 0.059 & 0.049 & 0.047 \\
\hline 530 & 0.058 & 0.049 & 0.048 \\
\hline
\end{tabular}




\begin{tabular}{|c|c|c|c|}
\hline 531 & 0.055 & 0.048 & 0.047 \\
\hline 532 & 0.057 & 0.048 & 0.047 \\
\hline 533 & 0.057 & 0.049 & 0.047 \\
\hline 534 & 0.058 & 0.048 & 0.047 \\
\hline 535 & 0.058 & 0.048 & 0.047 \\
\hline 536 & 0.055 & 0.047 & 0.046 \\
\hline 537 & 0.056 & 0.048 & 0.047 \\
\hline 538 & 0.055 & 0.047 & 0.045 \\
\hline 539 & 0.057 & 0.047 & 0.047 \\
\hline 540 & 0.055 & 0.046 & 0.044 \\
\hline 541 & 0.054 & 0.045 & 0.045 \\
\hline 542 & 0.055 & 0.045 & 0.046 \\
\hline 543 & 0.055 & 0.047 & 0.045 \\
\hline 544 & 0.055 & 0.045 & 0.045 \\
\hline 545 & 0.056 & 0.045 & 0.045 \\
\hline 546 & 0.056 & 0.046 & 0.045 \\
\hline 547 & 0.054 & 0.046 & 0.045 \\
\hline 548 & 0.053 & 0.045 & 0.043 \\
\hline 549 & 0.055 & 0.045 & 0.045 \\
\hline 550 & 0.056 & 0.046 & 0.047 \\
\hline 551 & 0.055 & 0.045 & 0.044 \\
\hline 552 & 0.053 & 0.044 & 0.044 \\
\hline 553 & 0.053 & 0.045 & 0.044 \\
\hline
\end{tabular}




\begin{tabular}{|r|r|r|r|}
\hline 554 & 0.052 & 0.044 & 0.044 \\
\hline 555 & 0.053 & 0.045 & 0.045 \\
\hline 556 & 0.054 & 0.044 & 0.043 \\
\hline 557 & 0.053 & 0.044 & 0.042 \\
\hline 558 & 0.053 & 0.045 & 0.043 \\
\hline 559 & 0.053 & 0.045 & 0.043 \\
\hline 560 & 0.054 & 0.044 & 0.043 \\
\hline
\end{tabular}

The resultant spectrum in the region NPs would be expected to have a max absorption appeared to show no sign of NPs and mirrored the spectrum of acetone alone (Figure 7 and Figure 8). An excerpt of the raw data (Table 3) displayed a small maximum difference between fresh acetone and the left-over sample acetone of ---. The evidence suggested that it was not likely NPs were dislodged from the surface of GAC, further contaminating the water and that TCE degradations rates from these samples may be resultant from NPs adsorbed onto GAC and degraded by the NPs.

\subsection{Effect of Synthesis Time}

In previous experiments, the NP samples were allowed to form under 24-hour periods. This time was chosen for experimental convenience. Here, the effect of formation time on GAC supported Pd/Au NPs catalyst to potentially expedite the synthesis process was investigated. Samples were synthesized in one-hour intervals between 0 and 24 hours and here the 1-hour and 24-hour samples are compared. 
The images in Figure 9 and Figure 11 show Pd and Au NPs supported by graphene.

Graphene was used as a support in the place of the denser, thicker GAC, for better characterization using TEM. Figure 9 shows a sample that was fabricated over 24 hours and a cluster of Pd NPs surrounding an Au core.
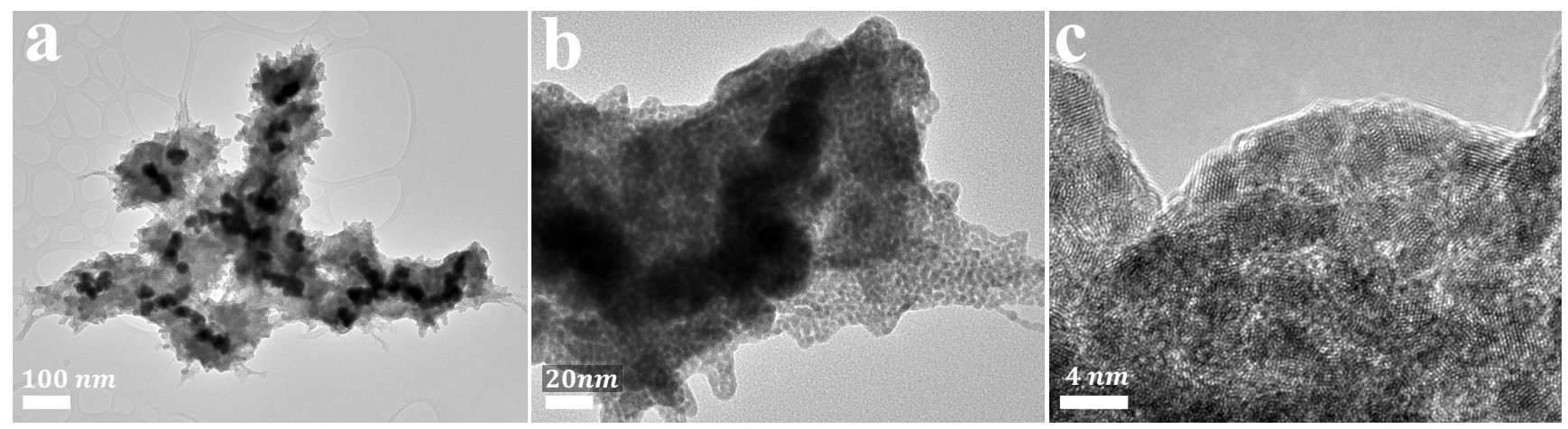

Figure 9: (a). Low magnification TEM images of Pd/Au NPs on graphene. (b) Higher magnification showing a core-shell structure. (c) NPs are crystalline. This sample had a 24-hour synthesis. A large cluster of Pd NPs surround Au core.

This structure is confirmed by STEM dark-field (Figure 10). The structure is consistently found throughout the surface of graphene support. These NPs resemble the expected structure of $\mathrm{Pd} / \mathrm{Au}$ NPs [3][4]. 

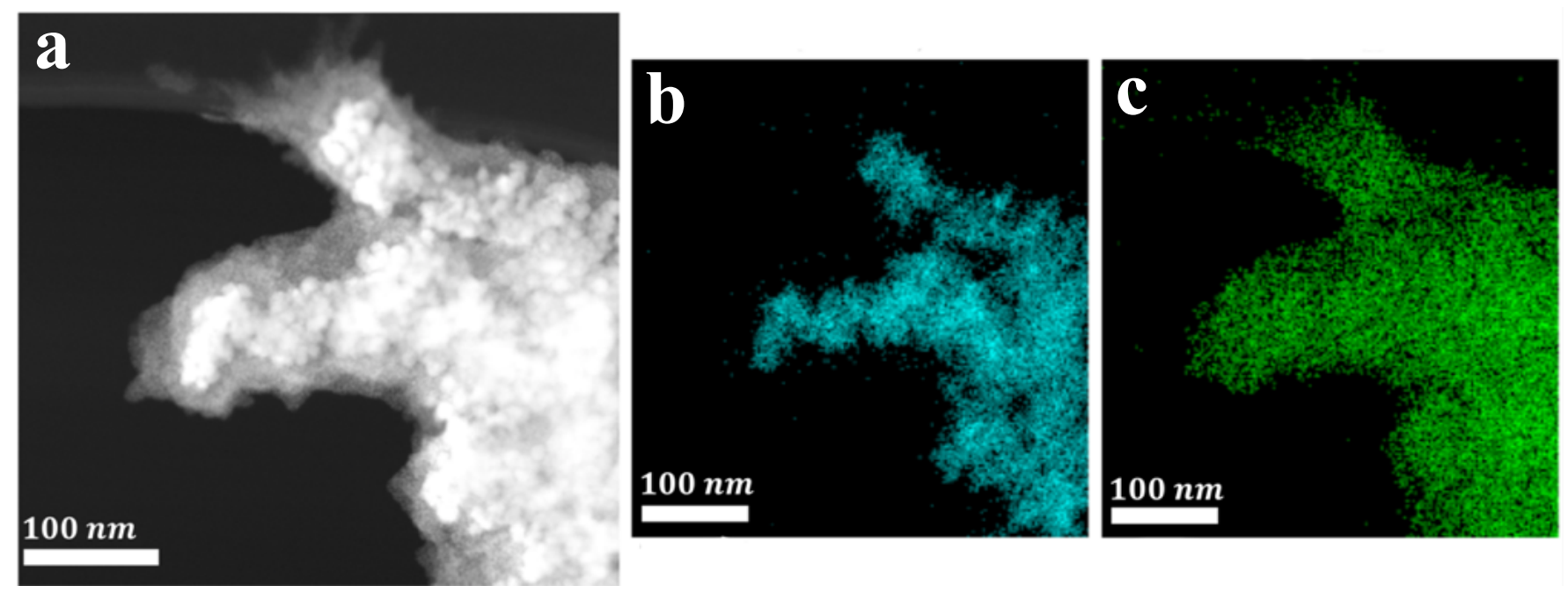

Figure 10: (a) STEM dark-field image with EDS maps of (b) Pd (green) and (c) Au (blue). This confirms Pd shell Au core structure of the cluster.

Likewise, after only 1 hour of synthesis (Figure 11), these similar clusters were observed (Figure 11b). In addition, the sample also contains what appears to be "less developed" NPs (Figure 11c) which have not gro $n$ to the size of the larger NPs in Figure 9b. This $\mathrm{Pd}_{\text {shell }} \mathrm{Au}_{\text {core }}$ structure appears to be the same; however, the Pd coating is attenuated.

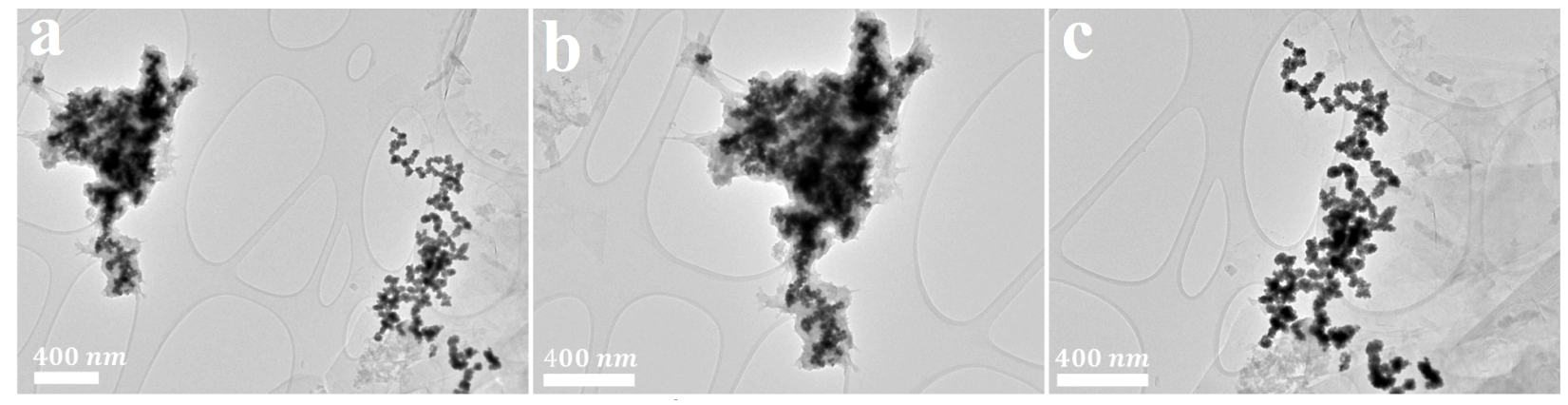

Figure 11: (a). Low magnification TEM images of Pd/Au NPs on graphene. While some NP clusters are fully formed (b), others have a thinner, not fully formed, Pd coating (c).

For father characterization, both samples were synthesized without the support and isolated in DI-water for UV-vis analysis. A higher absorption for Pd/Au NPs were found in the 24-hour sample compared with the 1-hour sample (Figure 12), observed at the expected peak 
around 500-600nm [6]. This suggests that despite an incomplete formation of the cluster at $1 \mathrm{hr}$, the catalyst still contains $\mathrm{Pd} / \mathrm{Au}$ NPs to degrade TCE.

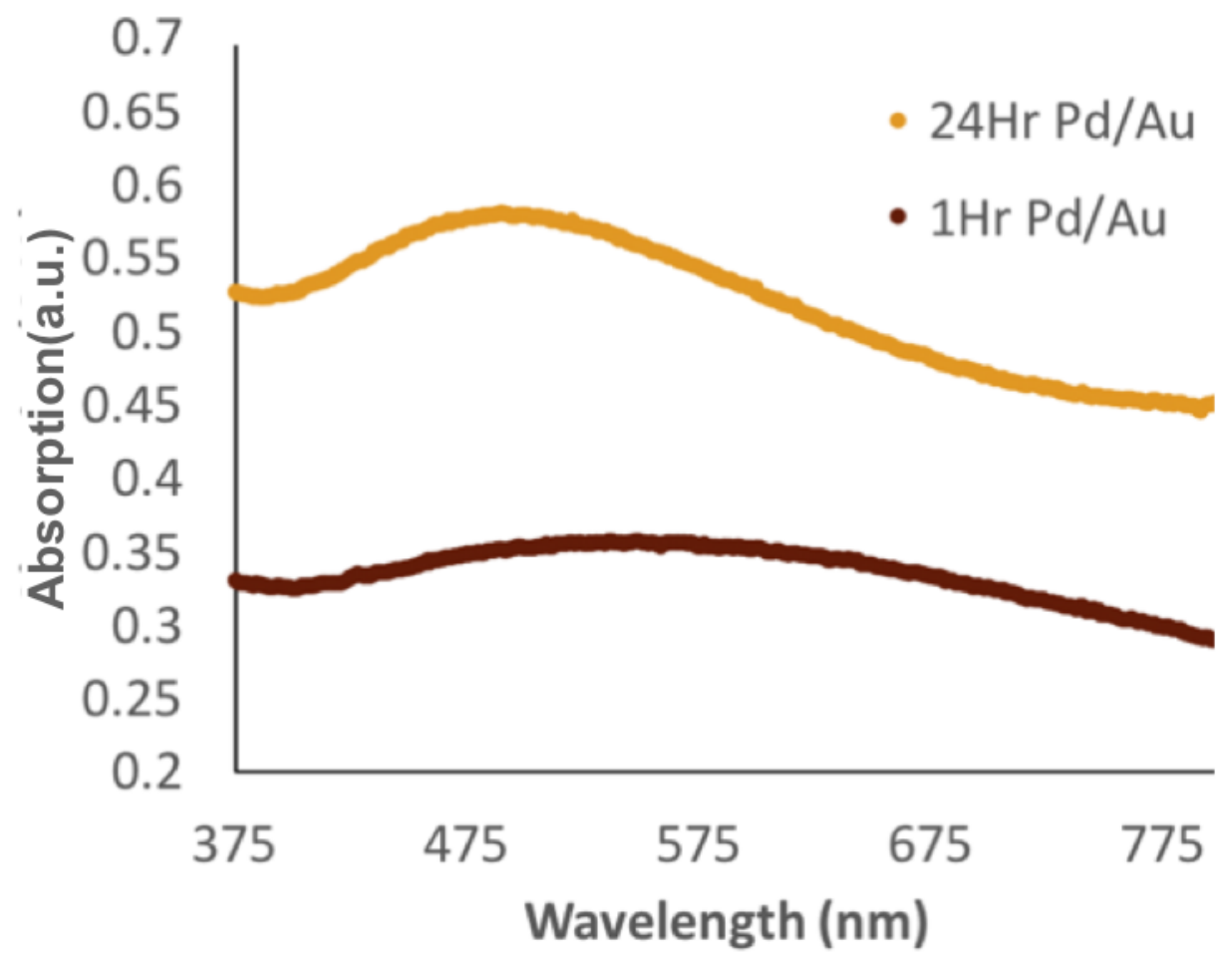

Figure 12: UV-Vis analysis shows absorption is higher when NPs are given longer to react

The1-hour sample differs from the 24-hour sample in that some of the NPs on their carbon support do not fully form in only 1 hour. With an additional 23 hours, the NP clusters grow in size and as a result, loses surface area that is able to react with any surrounding contaminate. This could result in a 1-hour sample potentially be capable of degrading TCE and other contaminants even more efficiently than the 24-hour sample. Since the Pd loading is kept consistent with commercial catalysts, the addition of Au using this synthesis process appears to produce a more potent catalyst. In conclusion, when $\mathrm{Au}$ is used as a promoter, lowering the $\mathrm{Pd}$ loading does not compromise the activity and performance of the catalyst even though a shorter synthesis causes the NPs to be smaller. That is, while synthesis time effects the size of the $\mathrm{Pd} / \mathrm{Au}$ NPs cluster, it does not affect the performance of the catalyst. In addition, this investigation also 
helped answer the question about when nanoparticles formed in the synthesis process. The size of the nanoparticles seemed directly related to the amount of time they were left to react, so one may assume that the synthesis of nanoparticles takes place during this reaction time. To further examine the performance of the catalyst, a systematic analysis of TCE degradation using catalysts made over different synthesis times is currently being tested using gas chromatography. Preliminary results are in agreement with the results presented here. 


\section{Conclusion}

This thesis discusses the optimization of the novel solvothermal, green synthesis process of $\mathrm{Pd} / \mathrm{Au}$ carbon supported nanoparticles (NPs) devolved by the Jiao Group at Portland State University. The pre-sonication of Au precursor, washing of the carbon supported NP samples, and reaction time of the samples were explored. These experiments encapsulate a small part of PhD Candidate, Kavita Meduri's, dissertation.

The experimental results showed that pre-sonicating the Au precursor did not, in and of itself, synthesize nanoparticles regardless of the time it was pre-sonicated or how much Au precursor was added. Though the pre-sonication did not seem to synthesize the NPs, it may be possible that the pre-sonication is necessary for NPs to form later. This will need to be investigated in more detail in other studies.

Washing the catalyst seemed to be an effective method of ensuring NPs were not dislodge from the surface of the GAC. This could suggest that the degradation of TCE occurred both because of GAC's adsorptive surface and the catalytic nature of Pd/Au NPs. In addition, it may ensure that the water the $\mathrm{Pd} / \mathrm{Au}$ samples are meant to purify are not further contaminating the water.

The time the samples are allowed to react seem affect the size of the Pd/Au NPs cluster, but not the performance of the catalyst. This could help expedite the synthesis process while maintaining the effectiveness of the catalyst.

The preliminary results gathered here contribute to the understanding of the GAC-supported $\mathrm{Pd} / \mathrm{Au}$ NP catalysts. This research process allows the undergraduate student to gain significant research experience and problem-solving skill. 


\section{Appendix}

\&Matlab script to analyze the NPs dislodged from surface of GAC

close all

clear all

ঃDay 1

\%Read in raw data files

filename $=$ 'NP. $\mathrm{xlsx}$ ';

sheet $=1$;

$\mathrm{a}=$ 'B3:B603';

D1A1=xlsread ('NP.xlsx', sheet, a );

$\mathrm{b}=\mathrm{C} 3: \mathrm{C} 603^{\prime}$;

D1A2 $=x$ lsread ( 'NP. $x 1 s x$ ', sheet, $b)$;

$\mathrm{C}=$ ' D3 : D603' ;

D1B1=xlsread ( 'NP.xlsx', sheet, c );

$\mathrm{d}=$ 'E3:E603';

D1B2 $=x 1$ sread ( 'NP.xlsx', sheet, d) ;

$\mathrm{e}=$ ' K3 : K503' ;

$P=x l$ sread ('NP. $x l s x$ ', sheet, e)

$\mathrm{f}=$ 'L3:L603' ;

$A=x l$ sread ( 'NP. $x l s x '$, sheet,$f)$

¿plot data

subplot $(2,1,1)$

plot (D1A1)

hold on

plot (D1A2)

hold on

plot (D1B1)

hold on

plot (D1B2)

hold on

plot (P)

hold on

$\operatorname{plot}(A)$

hold off

\% Set limits on graph

$\mathrm{x} \lim \left(\left[\begin{array}{ll}410 & 500\end{array}\right]\right)$;

$y \lim \left(\left[\begin{array}{ll}0 & 2.5\end{array}\right]\right)$;

xlabel ('Wavelength')

ylabel ('Absorption (a.u)')

title('Day 1 Trials: Wavelenth Absorbed on Washed Acetone')

legend('A1', 'A2' ', B1', 'B2', 'NP Control', 'Acetone Control')

title(legend,' Trials: A\&B=Sample\# 1\&2=Wash\#' ) 


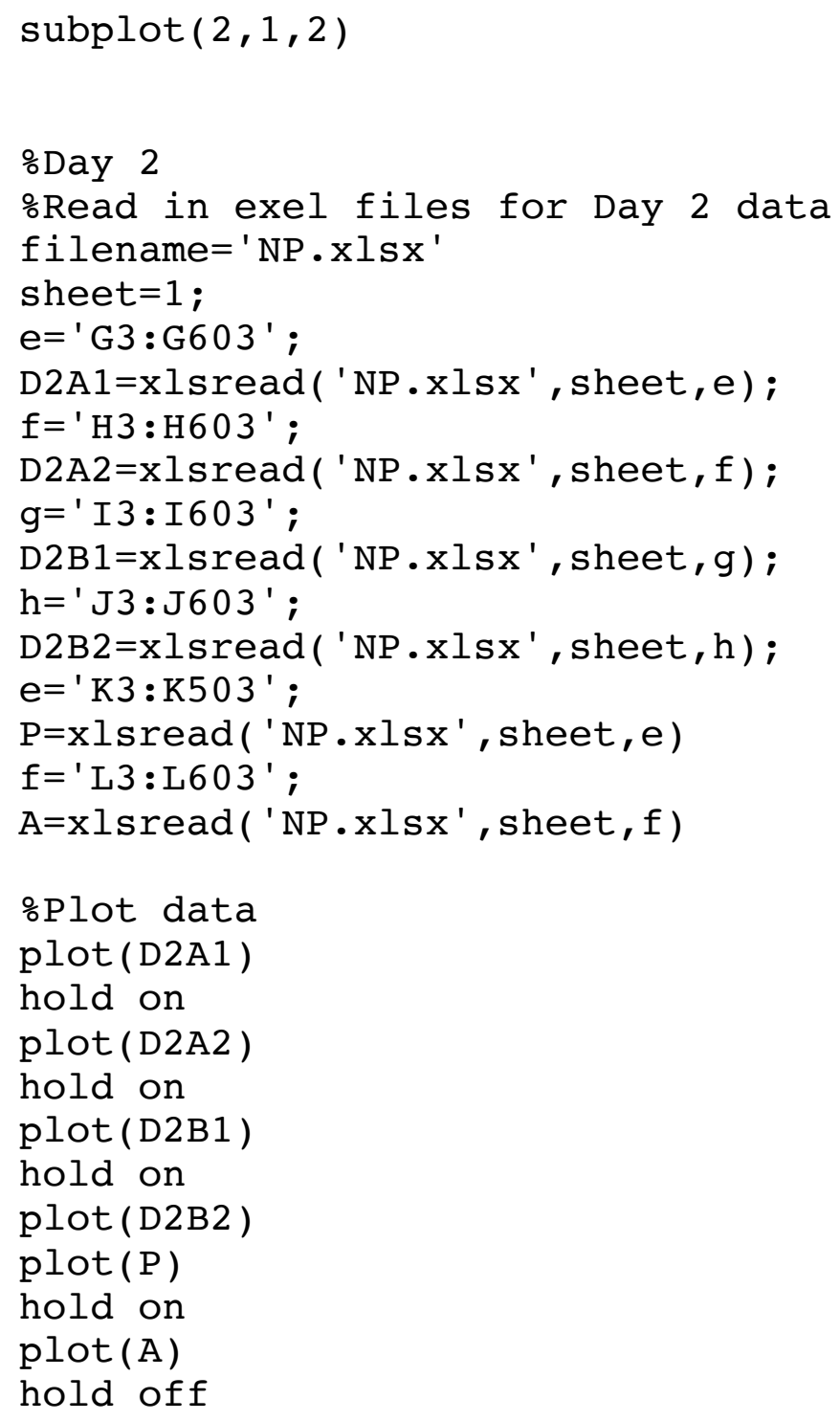




\section{Acknowledgments}

These studies are supported in part by NSF awards No. 1507707 and No. 1560383, and NIH Build EXITO program. Special thanks for the contributions and help from Ph.D. Candidate, Kavita Meduri and Professor, Jun Jiao for their guidance and the opportunity to conduct this research with her group. 


\section{References}

[1] Bauman Angela, Page Elena, Mueller Charles, Burr Greg, Hitcock Edward. Evaluation of Neurological Dysfunction Among Works Exposed to Trichloroeythylene: Hyattsville, MD: National Center for Health Statistics. March 2008 https://www.cdc.gov/niosh/hhe/reports/pdfs/2004-0372-3054.pdf

[2] Brooks, D., R. R. Roll, and W. Naylor. "Wastewater technology fact sheet granular activated carbon adsorption and regeneration." Environmental Protection Agency, USA 832 (2000).

[3] Chaplin, Brian P., Martin Reinhard, William F. Schneider, Christoph Schüth, John R. Shapley, Timothy J. Strathmann, and Charles J. Werth. "Critical review of Pd-based catalytic treatment of priority contaminants in water." Environmental science \& technology 46, no. 7 (2012): 3655-3670.

[4] Eustis, Susie, and Mostafa A. El-Sayed. "Why gold nanoparticles are more precious than pretty gold: noble metal surface plasmon resonance and its enhancement of the radiative and nonradiative properties of nanocrystals of different shapes." Chemical society reviews 35, no. 3 (2006): 209-217.

[5] Lin, X. M., C. M. Sorensen, and K. J. Klabunde. "Ligand-induced gold nanocrystal superlattice formation in colloidal solution." Chemistry of materials 11, no. 2 (1999): 198-202.

[6] McAllister, Michael J., Je-Luen Li, Douglas H. Adamson, Hannes C. Schniepp, Ahmed A. Abdala, Jun Liu, Margarita Herrera-Alonso et al. "Single sheet functionalized graphene by oxidation and thermal expansion of graphite." Chemistry of materials 19, no. 18 (2007): 43964404.

[7] McKinnon, Ronald J., and John E. Dyksen. "Removing Organics From Groundwater Through Aeration Phs GAC." (1984).

[8] Meduri, Kavita, Andrew Barnum, Graham O'Brien Johnson, Paul G. Tratnyek, and Jun Jiao. "Characterization of Palladium and Gold Nanoparticles on Granular Activated Carbon as an Efficient Catalyst for Hydrodechlorination of Trichloroethylene." Microscopy and Microanalysis 22 (2016): 332.

[9] Meduri, Kavita, Stauffer, Candice, Linder, Thomas, Johnson, Graham O'Brien, Tratnyek, Paul G, and Jiao Jun. "Effect of Synthesis Temperature on the Formation of GAC supported Pd and Au NPs." Microscopy and Microanalysis 23, no. S1 (2017): 1916-1917.

[10] Meduri, Kavita; Stauffer, Candice; Qian, Wen; Zietz, Otto; Barnum, Andrew; O’Brien Johnson, Graham; Fan, Dimin; Ji, Weixiao; Zhang, Changwen; Tratnyek, Paul; Jiao, Jun. "Palladium and Gold Nanoparticles on Carbon Supports as Highly Efficient Catalysts for Effective Removal of Trichloroethylene." Journal of Materials Research (2018) 
[11] Scarpa, F., S. Adhikari, and A. Srikantha Phani. "Effective elastic mechanical properties of single layer graphene sheets." Nanotechnology 20, no. 6 (2009): 065709.

[12] Siebert, Stefan, Jacob Burke, Jean-Marc Faures, Karen Frenken, Jippe Hoogeveen, Petra Döll, and Felix Theodor Portmann. "Groundwater use for irrigation-a global inventory." Hydrology and Earth System Sciences 14, no. 10 (2010): 1863

[13] Stauffer, Candice, Meduri, Kavita, Johnson, Garaham O’brien, Tratnyek, Paul, and Jiao, Jun "Effect of Synthesis Time of Carbon Supported Pd/Au NPs on TCE Degradation Effect of Synthesis Time of Carbon Supported Pd/Au NPs on TCE degradation" Microscopy and Microanalysis 24, (2018) 